\title{
离轴非球面 $\mathrm{SiC}$ 反射镜的精密铣磨加工技术
}

\author{
张志宇 李锐钢 郑立功 张学军 \\ (中国科学院长春光学精密机械与物理研究所中国科学院光学系统 \\ 先进制造技术重点实验室 长春 130033)
}

\begin{abstract}
摘要: 离轴非球面反射镜是高分辨率、大视场空间相机的核心元件, 其镜面的形状精度和表面质量要求极高, 加工难度很大, 一直是光学系统先进制造技术的瓶颈。针对国家对空间大型 $\mathrm{SiC}$ 光学元件制造技术的重大需求，开展以大口径、高陡度、大 离轴量和大偏离量为特点的离轴非球面反射镜的精密铣磨加工技术研究。开发非球面反射镜计算机辅助数控编程技术, 提出 五轴联动斜轴定角度加工方式, 建立旋转中心在镜面外的螺旋加工轨迹, 避免镜面几何中心处的加工残留。并基于五轴加工 中心(型号 DMG Ultrasonic 100-5) 搭建超声振动辅助铣磨加工平台, 以 $900 \mathrm{~mm} \times 660 \mathrm{~mm}$ 口径离轴非球面 SiC 反射镜镜坏为 加工样件进行工艺试验, 加工面形精度峰谷值(Peak to valley, PV)达到 $18.8 \mu \mathrm{m}$, 方均根值(Root mean square, RMS)达到 $3.5 \mu \mathrm{m}$ 。 研究为按照非球面方程高精度铣磨超大尺度精度比离轴非球面反射镜提供了有效的解决方案。
\end{abstract}

关键词: 离轴非球面 $\mathrm{SiC}$ 反射镜 精密磿削 $\mathrm{CAD} / \mathrm{CAM}$ 加工轨迹优化 超声振动辅助加工

中图分类号: TG58

\section{Precision Grinding Technology for the Off-axis Aspherical Silicon Carbide Mirror Blank}

\section{ZHANG Zhiyu LI Ruigang ZHENG Ligong ZHANG Xuejun}

(Key Laboratory of Optical System Advanced Manufacturing Technology, Changchun Institute of Optics,

Fine Mechanics and Physics, Chinese Academy of Sciences, Changchun 130033)

\begin{abstract}
Off-axis aspheric mirrors are the key components in a high-resolution, large field-of-view space camera, and are very hard to machine due to extremely strict requirements on shape accuracy and surface quality. Therefore it is a bottleneck of the advanced optical manufacturing technology. Computer-aided NC programming techniques for mirror blanks with large-diameter, high-steepness, large-deviation, and large off-axis-amount are developed. An oblique five-axis processing method with a decenter spiral tool path is proposed to avoid the residual shape error in the geometric center of mirror blank. Based on a five-axis ultrasonic vibration-assisted machining center, a $900 \mathrm{~mm} \times 660 \mathrm{~mm}$ diameter off-axis aspheric SiC mirror blank is successfully machined, obtaining a surface accuracy of $18.8 \mu \mathrm{m}$ peak to valley (PV), and $3.5 \mu \mathrm{m}$ root mean square (RMS). Effective and precision machining technologies in accordance with the equation of the off-axis aspherical mirrors with huge scale-precision ratio are provided.
\end{abstract}

Key words: Off-axis asphere $\mathrm{SiC}$ mirror Precision grinding CAD/CAM Tool path optimization

Ultrasonic-vibration assisted machining

\section{0 前言}

环绕地球轨道运行的空间相机担负着空间对 地观测、深空探测等重任。早期的空间相机采用同 轴反射式光学系统成像, 例如著名的哈勃太空望远

* 国家重点基础研究发展计划(973 计划, 2011CB013200)和国家自然科学 基金(61036015)资助项目。20121029 收到初稿, 20130227 收到修改稿
镜。近年来建造的空间相机多采用三反消像散系 统 ${ }^{[1]}$ 。该系统由三片离轴非球面反射镜构成, 可以 同时实现高分辨率与大视场, 没有中心遮拦, 传递 函数高, 成为当代宽幅详查空间相机普遍采用的光 学设计。为获得高清图像, 反射镜的尺寸很大(有效 口径可达 $1 \mathrm{~m}$ 以上), 且有效口径内镜面形状精度方 均根值(Root mean square, RMS)值要求达到 $\lambda / 50$ $(\lambda=632.8 \mathrm{~nm})$, 相当于 $12 \mathrm{~nm}$ 。尺度精度比高达 $10^{8}$, 
因而加工难度非常大。离轴非球面反射镜的制造水 平是一个国家光学制造能力的重要体现。

离轴非球面反射镜的加工主要包含四个工艺 过程: 毛坏制备、铣磨加工、精密研磨和精密抛光。 近年来国内在精密研磨、精密抛光方面的研究成果 丰富, 但在铣磨加工阶段, 一般还是按照离轴非球 面的最接近球面进行加工。最接近球面铣磨完成后, 依靠精密研磨、精密抛光等工序, 多周期修正最接 近球面与离轴非球面的偏差量, 直至最终达到图纸 设计要求。铣磨最接近球面的方法简便易行, 但有 时离轴非球面的表面形状与最接近球面的偏差量很 大, 可能在数百微米、甚至到毫米量级。通过研磨 手段去除如此大的偏差量, 需要很长时间(几个月甚 至几年)。例如, 法国 REOSC 空间光学制造中心加 工的 $8.2 \mathrm{~m}$ 口径大望远镜的主镜, 设计非球面与最 接近球面的最大矢高差为 $2.3 \mathrm{~mm}$, 这导致需要去除 的工件材料高达 $150 \mathrm{~kg}^{[2]}$ 。如果能够按照离轴非球 面的设计形状进行铣磨, 则后续精密研磨只需要修 正铣磨加工的误差, 因而可大幅度缩短整个反射镜 的加工时间。

在离轴非球面铣磨加工方面, 英国的 Cranfiled 精密工程研究所 ${ }^{[3]}$ 的工作较为突出。他们研制的 Big Optix $\left(\mathrm{BOX}^{\circledR}\right)$ 大型 $\mathrm{CNC}$ 超精密磨床, 在加工对角 $1.45 \mathrm{~m}$ 六角形口径 Zerodur 材料离轴非球面时, 仅 用 $20 \mathrm{~h}$ 就去除 $1 \mathrm{~mm}$ 的加工余量, 获得峰谷值(Peak to valley, PV)优于 $5.5 \mu \mathrm{m}$ 的形状精度。BOX ${ }^{\circledR}$ 机床 性能优异, 但铣磨 $\mathrm{SiC}$ 时, 砂轮磨损依然非常严重, 以至于每 $26.5 \mathrm{~min}$ 就要修整一次砂轮以保证加工力 小于 $100 \mathrm{~N}^{[4]}$ 。 $\mathrm{BOX}^{\circledR}$ 机床属于三轴机床, 自由度少, 当加工高陡度离轴非球面时并不具备优势。另外, 该机床开发成本昂贵，性价比并不高。

国内在中小口径非球面铣磨研究方面也取得 了显著成果。成都精密光学工程研究中心 ${ }^{[5]}$ 使用引 进的 MFP-125 成形磨床, 开展 $430 \mathrm{~mm} \times 430 \mathrm{~mm}$ 口 径熔石英方形非球面镜的铣磨加工研究, 得到面形 精度 PV 为 $4.2 \mu \mathrm{m}$, 表面粗粘度 $R_{\mathrm{a}}$ 为 $0.1 \mu \mathrm{m}$ 的结 果。国防科技大学 ${ }^{[6-7]}$ 使用自研的 AOCMT 光学加工 机床, 采用恒去除量磨削方式, 实现了 $\Phi 210 \mathrm{~mm}$ 口径 $\mathrm{K} 9$ 玻璃抛物面的加工, 获得的面形精度 $\mathrm{PV}$ 值达到 $4.8 \mu \mathrm{m}$ 。

国内外报道的铣磨加工, 对象多为中小口径 $(<500 \mathrm{~mm})$ 的同轴非球面, 或是面形平缓的离轴非 球面。对于大口径、大偏离量和高陡度的离轴非球 面元件的研究相对较少。另外, 国内外对 Zerodu 和 ULE 材料研究较多, 对 $\mathrm{SiC}$ 材料反射镜的铣磨加 工问题缺乏深入了解。随着反射镜尺寸不断增大,
与 Zerodu 和 ULE 相比, $\mathrm{SiC}$ 材料的性能优势越来 越明显 ${ }^{[8]}$, 有望成为未来空间相机反射镜的主要材 料。但是, $\mathrm{SiC}$ 是仅次于金刚石和 $\mathrm{CBN}$ 的超硬脆性 材料, 莫氏硬度高达 9.3。采用传统设备和工艺加工 $\mathrm{SiC}$ 反射镜, 材料去除率低、砂轮磨损剧烈并由此 导致加工精度严重恶化, 加工难度很大。因而急需 研究 $\mathrm{SiC}$ 反射镜的高效高精度加工技术。

本文面向国家对空间大型 $\mathrm{SiC}$ 反射镜制造技术 的重大需求, 致力于研究和开发超大尺度精度比离 轴非球面反射镜的铣磨加工技术。开发计算机辅助 数控编程技术, 提出五轴联动斜轴定角度加工方式, 并给出消除工件几何中心处加工残留的方法。最后 在超声振动辅助五轴加工中心上, 成功开展了某离 轴非球面 $\mathrm{SiC}$ 反射镜的铣磨加工试验。

\section{1 离轴非球面 CAD 理论模型的建立}

\section{1 离轴非球面的概念}

如图 1 所示, 离轴非球面是按照给定几何形状 及尺寸从回转对称的非球面(称为母镜)上截取下的 一部分。图 1 中 $O$ 为母镜的几何中心, $O^{\prime}$ 为离轴非 球面的几何中心, $z$ 轴方向为光轴方向。离轴非球 面沿 $x$ 轴(母线)方向偏离了母镜的几何中心。该偏 离量称为离轴量。有时, 出于镜坏制备和加工便利 的考虑, 需要将离轴非球面沿着 $y$ 向对称轴进行旋 转, 以获得近轴边与远轴边高度相同的镜面, 该旋 转角度可视为离轴角。因此, 离轴非球面无法用解 析表达式进行描述, 本身也不再具有回转对称特性, 是一种典型的自由曲面光学元件。

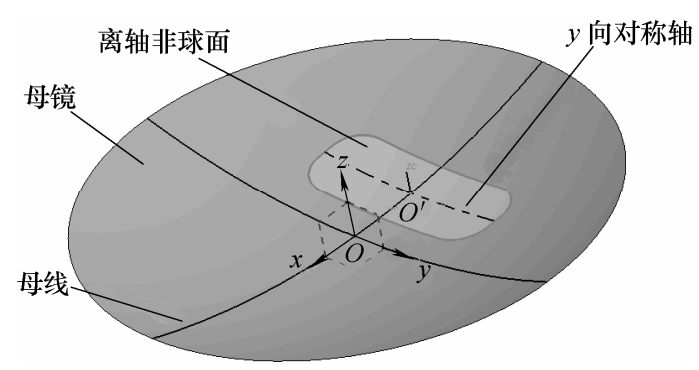

图 1 离轴非球面的示意图

离轴非球面的母镜为回转对称的同轴非球面。 设回转对称轴(光轴)为 $z$ 轴, 以非球面的顶点作为 坐标原点, 则非球面方程可表述为 ${ }^{[9]}$

$$
\begin{gathered}
z(S)=\frac{c S^{2}}{1+\sqrt{1(1+k) c^{2} S^{2}}}+A_{2} S^{4}+A_{3} S^{6}+A_{4} S^{8}+ \\
A_{5} S^{10}+A_{6} S^{12}+A_{7} S^{14}+A_{8} S^{16}+\cdots
\end{gathered}
$$

式中, $S^{2}=x^{2}+y^{2}, c=1 / R$ ( $R$ 为顶点曲率半径), $k$ 为 二次曲面常数, $k=-e^{2}$ ( $e$ 为二次曲面的偏心率), $A_{i}$ 
$(i=2,3, \cdots)$ 为非球面变形系数, 也称为高次项系数。 如果 $A_{i}$ 全部为零, 则非球面为一个二次曲面。

本文提出基于 $\mathrm{CAD} / \mathrm{CAM}$ 技术, 按照上述非球 面方程直接铣磨离轴非球面反射镜的新方法。首先 需要在 $\mathrm{CAD}$ 软件中建立离轴非球面模型。CAD 模 型一经建立, 就可以利用 CAM 技术规划刀具路径、 自动生成加工代码, 最后使用相应机床进行加工。

\section{2 离轴非球面 CAD 模型的建立}

$\mathrm{UGNX}$ 软件是商业化 $\mathrm{CAD}$ 软件的杰出代表。 基于该软件, 可以输入曲线方程直接建立曲线模型, 但不能输入曲面方程以建立曲面模型。经过调查, 未发现商业化的 CAD 软件具有按照曲面方程直接 建立曲面模型的功能。本文开发通过非球面的母线 方程建立非球面解析模型的方法。

经数学推导后得知, 非球面的母线方程(子午剖 面方程)可以表述为下面的多项式形式

$$
\begin{gathered}
z(x)=B_{1} x^{2}+B_{2} x^{4}+B_{3} x^{6}+B_{4} x^{8}+B_{5} x^{10}+B_{6} x^{12}+ \\
B_{7} x^{14}+B_{8} x^{16}+\cdots
\end{gathered}
$$

式中, $B_{j}(j=1,2, \cdots)$ 为多项式系数, 并且式(1)、(2) 的系数组间存在着如下确定关系

$$
\begin{gathered}
B_{1}=\frac{1}{2} c=\frac{1}{2 R} \\
B_{2}=A_{2}+\frac{1}{8}(1+k) c^{3} \\
B_{3}=A_{3}+\frac{1}{16}(1+k)^{2} c^{5} \\
B_{4}=A_{4}+\frac{5}{128}(1+k)^{3} c^{7} \\
B_{5}=A_{5}+\frac{7}{256}(1+k)^{4} c^{9} \\
B_{6}=A_{6}+\frac{21}{1024}(1+k)^{5} c^{11} \\
B_{7}=A_{7}+\frac{33}{2048}(1+k)^{6} c^{13} \\
B_{8}=A_{8}+\frac{429}{32768}(1+k)^{7} c^{15}
\end{gathered}
$$

将已知系数的母线方程式(2)输入到 CAD 软件 (例如 UG NX), 就可以绘制出母线的模型。绕光轴 旋转母线后即可得到非球面模型。之后, 可根据离 轴非球面在垂直于光轴平面内的轮廓形状、离轴量 及离轴角等参数, 从非球面模型中截取出离轴非球 面的模型。

本文要加工的离轴非球面, $k=0.25, R=1400$ $\mathrm{mm}, A_{4}=-1.6 \times 10^{-12}$, 离轴量为 $365 \mathrm{~mm}$, 离轴角为 $16^{\circ}$ 。其沿光轴的投影为 $900 \mathrm{~mm} \times 660 \mathrm{~mm}$ 的圆角矩 形, 四个圆角半径均为 $300 \mathrm{~mm}$ 。图 2 为建立的镜 面 CAD 模型。由于该模型是严格按照离轴非球面 反射镜的参数建立的解析模型, 模型上任意点的坐 标值可以无限接近于理论值, 因此模型精度完全适 用于铣磨加工和超精密加工。

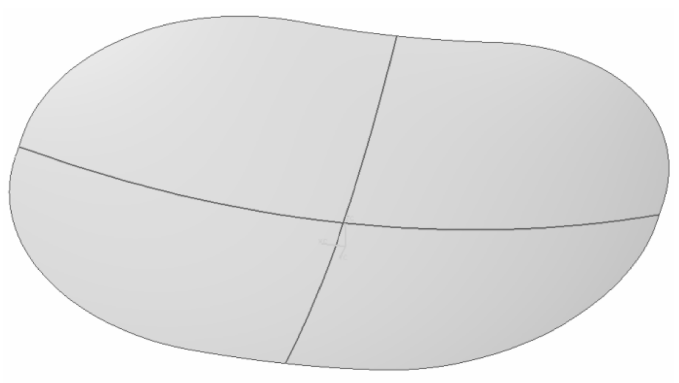

图 2 建立的离轴非球面 CAD 模型

经分析得知, 该离轴非球面的非球面度 (与最接 近球面的最大偏差量)为 $0.954 \mathrm{~mm}$, 陡度 $(R)$ 达到 1.25 , 综上可知, 该镜面的特点是大口径、大离轴 量、大非球面度和高陡度。

\section{2 离轴非球面的加工工艺}

\section{1 工件摆放位置}

回转对称非球面的常用加工方法如图 3 所示。 因镜面几何中心与转台旋转中心重合, 转台旋转的 同时, 工具(刀具或砂轮)沿着母线 $z(x)$ 由边缘进给到 中心, 形成螺旋线加工轨迹。图 4 给出了离轴非球 面的两种加工方式。加工离轴非球面时, 通常是将 工件摆放在如图 4a 所示的离轴位置, 使离轴非球面 的加工转变为回转对称非球面加工问题 ${ }^{[10]}$ 。这种方 法使用的轴数少, 有一定优势, 尤其适用于小离轴 量、小口径光学元件的加工。

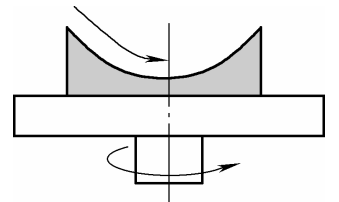

图 3 回转对称非球面镜坏摆放在同轴位置加工

但对大离轴量 $(>300 \mathrm{~mm})$ 及大口径 $(1 \mathrm{~m}$ 量级)光 学元件, 上述方法的劣势变得非常明显: 不仅浪费 机床空走刀时间, 而且要求机床必须具有大行程和 大转台, 这样的机床造价十分昂贵, 实际加工中很 难实现。为解决上述问题, 本文提出将工件摆放在 转台中心, 如图 $4 \mathrm{~b}$ 所示, 按照离轴非球面方程直接 进行加工。该加工方法不受离轴量大小的限制, 可 


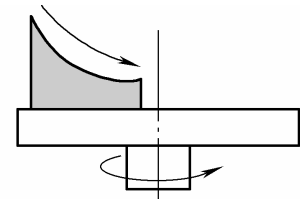

(a) 离轴位置

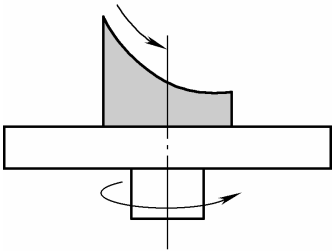

(b) 同轴位置

图 4 离轴非球面镜坏摆放位置与加工方式

最大程度增大机床可加工工件的尺寸 ; 由于不再具 有空走刀时间，因而加工时间大幅度减小。

\section{2 五轴联动斜轴定角度加工方式}

常见的磨削方式包括平行磨削, 横向磨削以及 斜轴磨削等。为加工小口径 $(<10 \mathrm{~mm})$ 高温模压成形 非球面模具, CHEN 等 ${ }^{[11}$ 提出了基于圆柱形砂轮的 三轴 $(x z B)$ 联动单点斜轴磨削方法, 如图 5 所示。砂 轮在沿 $x$ 向进给的同时, 还绕磨削点在 $O x z$ 平面内 摆动, 使工件表面磨削点处的法线与砂轮的轴线始 终保持固定角度 $\left(45^{\circ}\right)$ 。应用上述加工方式时, 砂轮 磨损虽然集中在一点, 但砂轮磨损引起的工件形状 误差可通过补偿加工消除。重要的是, 加工高陡度 非球面时, 该加工方法不会产生刀具与工件的碰撞 干涉, 因此优于 $\mathrm{BOX}^{\circledR}$ 机床使用的定轴变磨削点加 工方式 ${ }^{[12]}$ 。

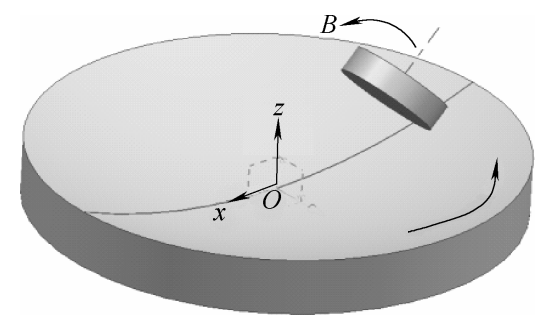

图 5 三轴联动单点斜轴加工方法

上述加工方式可加工回转对称非球面。对于图 6 所示的非回转对称离轴非球面而言, 因为工件表 面各点法线均不一致, 需要再增加一个轴( $y$ 轴)来调 整砂轮的径向坐标，使得工件表面磨削点处的法线 与砂轮的轴线保持固定角度。同时, 由于离轴非球 面不具有回转对称特性, 加工时需要转台有角度定 位功能( $C$ 轴)。综上所述, 加工系统应增加两个自 由度, 因此提出如图 6 所示的五轴联动 $(x y z B C)$ 斜轴 定角度加工方式。

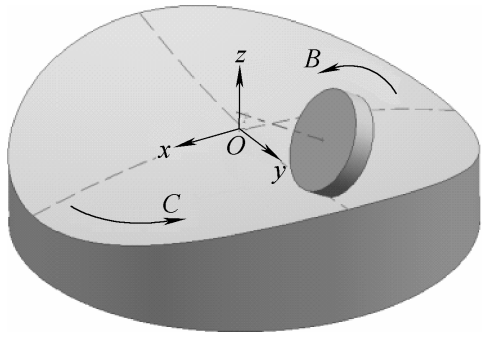

图 6 五轴联动斜轴定角度加工方法

\section{3 进给方式与加工效率}

需要强调的是, 当使用圆柱形砂轮进行加工 时, 可以通过合理选择砂轮进给方式提高加工效率。

图 5 所示的砂轮磨削方向与砂轮径向进给方向 垂直, 此时的加工表面如图 7a 所示。设砂轮轴与磨 削点处的法线夹角为 $\alpha\left(\right.$ 一般 $\left.\alpha=30 \sim 45^{\circ}\right)$, 进给量为 $f$, 则加工表面理论残留高度

$$
d_{\mathrm{p}}=f \sin \alpha \cos \alpha
$$

图 6 所示的砂轮磨削方向与砂轮径向进给方向 平行, 这时的加工表面如图 $7 \mathrm{~b}$ 所示。此时砂轮需要 沿着 $y$ 向进给，而非 $x$ 向。设砂轮的半径为 $R_{\mathrm{w}}$ (本 文 $\left.R_{\mathrm{w}}=100 \mathrm{~mm}\right)$, 则此时的加工表面理论残留高度 可近似为

$$
d_{\mathrm{c}}=\frac{f^{2}}{8 R_{\mathrm{w}}}
$$

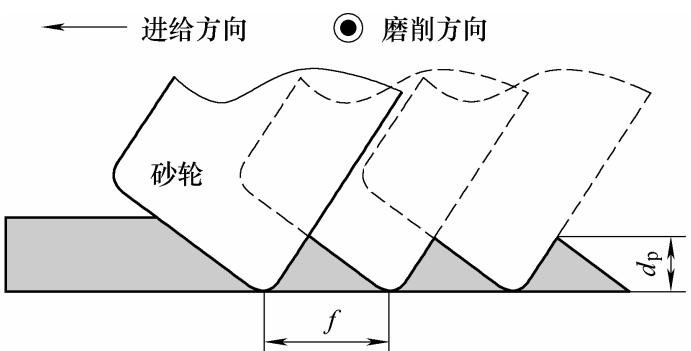

(a) 磨削方向与进给方向垂直

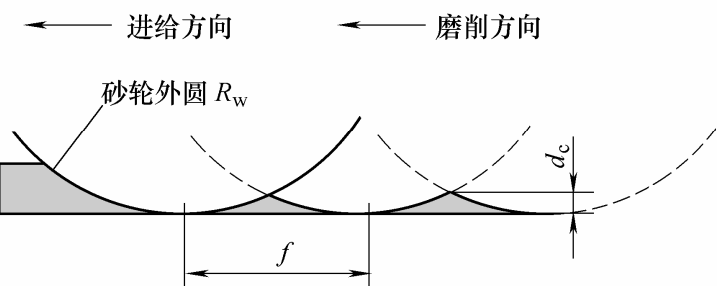

(b) 磨削方向与进给方向平行

图 7 加工表面残留进给痕与理论粗䊁度

实际加工中, 因 $R_{\mathrm{w}} \gg f$, 取相同 $f$ 值时, 则 $d_{\mathrm{c}} \ll d_{\mathrm{p}}$, 故应用图 6 所示的加工方式可显著降低表 面粗粗度。本文以获取形状精度为主要目的, 故大 幅度提高 $f$ 值以提高材料去除效率。这样带来的利 
好是加工时间缩短, 砂轮磨损降低, 被加工工件的 面形精度势必会得到提升。

\section{4 刀具轨迹优化}

五轴联动斜轴定角度加工方式可视为刀具角 度可变的慢刀伺服车削模式, 将在工件表面留下螺 旋线加工轨迹, 如图 8 所示。由于径向对刀误差不 可避免, 当采用螺旋线加工轨迹时, 总要在工件的 旋转中心处留下一个残留区域, 或是 $\Lambda$ 字形凸台, 或是 $\mathrm{V}$ 字形山坑。如果出现山坑形状, 将会大幅度 增加后续研磨余量。有关这一问题, 学者们进行了 相关研究 ${ }^{[13]}$ 。

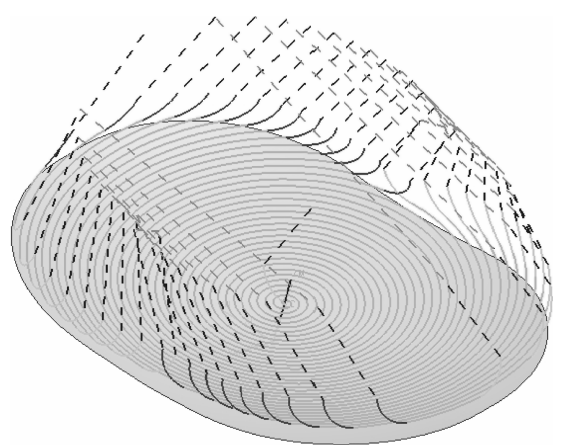

图 8 传统的螺旋线加工轨迹

上述工件中心处的加工残留问题可通过优化 刀具轨迹的方法解决。图 9 为提出的新加工轨迹。 依然采用螺旋线加工方式, 但是把螺旋中心设置在 镜面外。采用该轨迹加工出的工件表面不存在螺旋 中心, 因而也就不存在螺旋中心处残留的凹陷或者 凸台, 表面质量一致性好, 后期研磨工序去除量小。

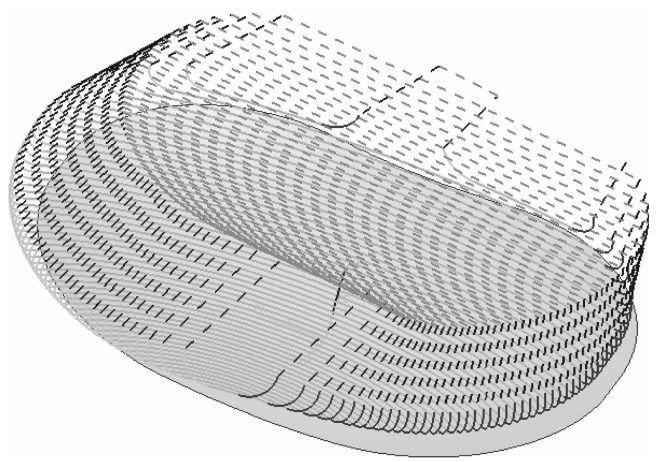

图 9 螺旋中心设置在镜面外的加工轨迹

\section{3 加工试验与精度测量}

\section{1 五轴联动超声振动加工中心}

五轴联动机床的加工自由度多, 能实现最优化 的加工策略, 包括刀具的路径选择、位姿调整和进 给方式等。因此, 本文采用德国引进的 $\mathrm{DMG}$ Ultrasonic 100-5 五轴联动加工中心进行加工。图 10
为该机床的结构示意图。该设备 $x y z$ 三轴行程均为 $1 \mathrm{~m}$, 编程分辨率为 $1 \mu \mathrm{m}$, 全行程定位精度为 $8 \mu \mathrm{m}$ 。 该设备为非正交机床, $B$ 轴为倾斜 $45^{\circ}$ 的摆头, 摆 动范围达 $-30^{\circ} \sim 180^{\circ}$, 可实现立卧加工转换。 $C$ 轴 为 $360^{\circ}$ 回转定位工作台。 $B C$ 两轴的角度分辨率为 $0.001^{\circ}$ 。图 11 为加工区域的照片。通过调整将镜面 的几何中心与转台旋转中心重合后, 使用定位板将 镜坏四个侧面固定。为提高 $\mathrm{SiC}$ 材料的去除效率, 并获得较高的表面质量, 还在该设备上配备了超声 主轴和超声振动发生器刀柄 ${ }^{[14]}$ 。同时, 超声振动辅 助加工减小了磨削力, 一定程度上弥补了五轴机床 刚度较低的不足。使用了树脂基金刚石杯形砂轮, 具体的试验条件如下表所示。

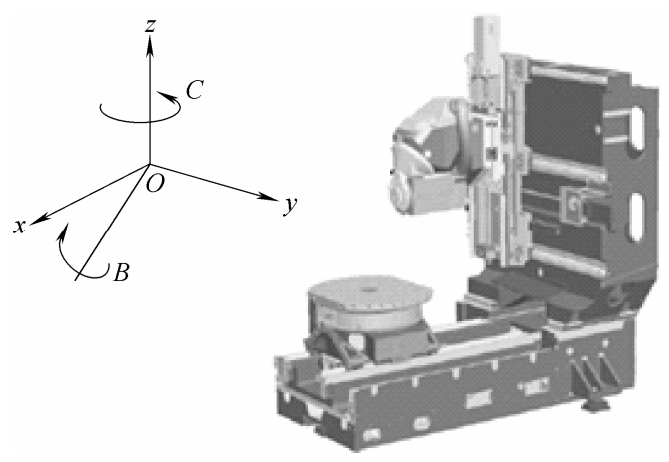

图 10 五轴联动超声振动加工中心结构示意图

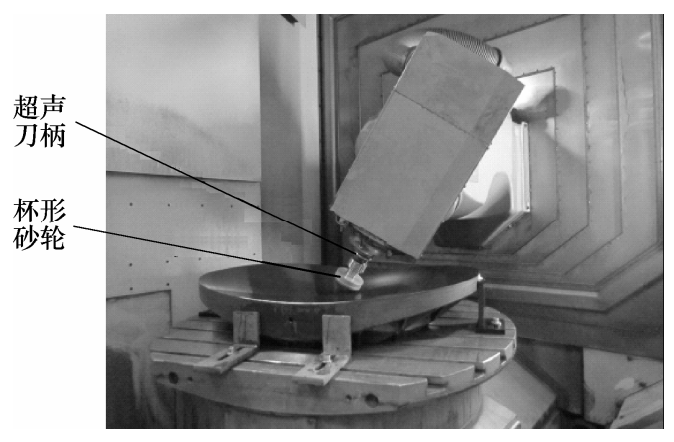

图 11 五轴联动超声振动加工中心外观图

\section{表 离轴非球面铣磨加工条件}

\begin{tabular}{lc}
\hline \multicolumn{1}{c}{ 项目名称 } & 型号或数值 \\
\hline 机床 & DMG Ultrasonic $100-5$ \\
砂轮 & 树脂基金刚石砂轮 \\
工件材料 & 反应烧结 $\mathrm{SiC}$ \\
直线工作台进给速度 $v /(\mathrm{mm} / \mathrm{min})$ & $1000 \sim 1500$ \\
磨削深度 $d / \mathrm{mm}$ & $0.2 \sim 0.5$ \\
径向进给量 $f_{\mathrm{r}} / \mathrm{mm}$ & $0.5 \sim 2.0$ \\
砂轮转速 $n /(\mathrm{r} / \mathrm{min})$ & $4000 \sim 6000$ \\
磨削液 & Blaser Swisslube Grindex \\
振动频率 $f_{\mathrm{v}} / \mathrm{kHz}$ & $20 \sim 30$ \\
\hline
\end{tabular}

\section{2 检测手段}

加工过程中, 通过机载 Renishow 测头在线测 
量工件形状, 并与 CAD 模型上相应测头接触点的 $z$ 坐标理论值进行比较, 从而获取镜面矢高误差分布 信息, 指导补偿加工。由于在线测量结果包含导轨 误差, 经过两次补偿加工后, 采用 ZEISS 公司生产 的三坐标测量机(型号 Prismo Navigator)测量了加工 表面形状误差分布, 如图 12 所示。加工表面的粗粘 度是通过白光干涉仪(型号 ZYGO NewView 7000) 测量相同条件下加工的小尺寸 $\mathrm{SiC}$ 试验块获得的。

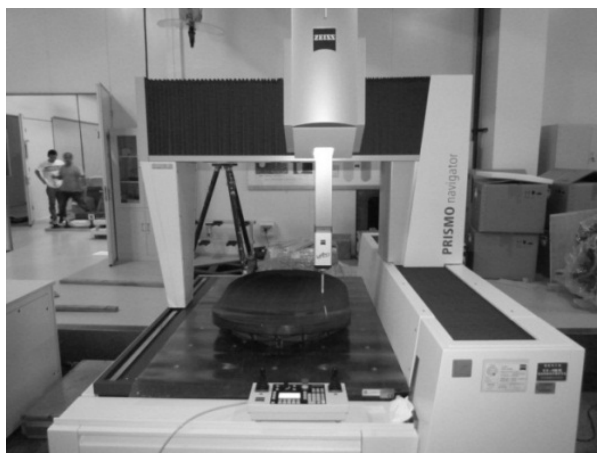

图 12 三坐标测量机外观图

\section{3 加工结果与讨论}

加工表面的形状误差分布如图 13 所示, 结果 $\mathrm{PV}$ 为 $18.8 \mu \mathrm{m}, \mathrm{RMS}$ 为 $3.5 \mu \mathrm{m}$ 。这一结果略逊于 $\mathrm{BOX}^{\circledR}$ 超精密机床的精加工水平，但明显优于其半 精加工水平 ${ }^{[15]}$ 。镜面两侧紧靠边缘部分局部有少许 较大误差, 为高点。理论上讲, 还可通过多周期补 偿加工使该面形误差收敛到 $10 \mu \mathrm{m}$ 以下，但耗时较 长, 因为该误差已接近机床的系统精度。应转入基 于应力盘的精密研磨工序, 面形误差收玫速度会相 对较快。

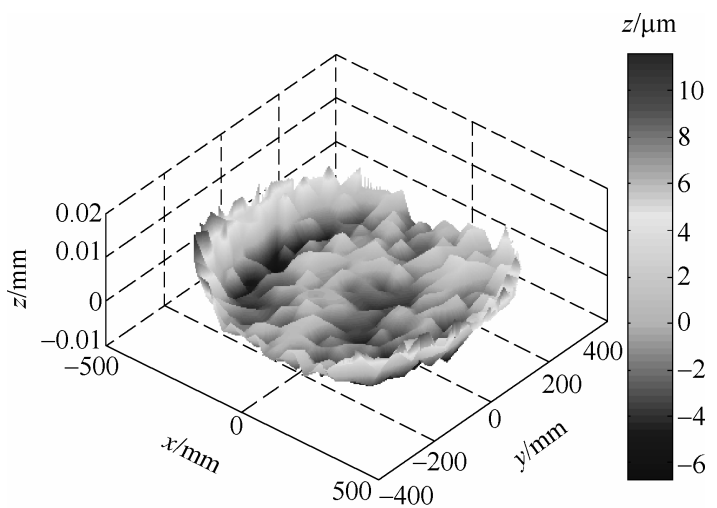

图 13 精密铣磨面形误差测量结果

加工表面上走刀痕迹清晰, 但无目视可见破 损。图 14 给出了表面粗糙度的测量结果, 其中 $\mathrm{PV}=10.521 \mu \mathrm{m}, R_{\mathrm{a}}$ 为 $524 \mathrm{~nm}$ 。图 14 中所示的横条 纹为转台每转一周砂轮的径向进给痕, 坚条纹为砂 轮自转一周的切向进给痕。表面粗粘度值较大的原 因是使用的砂轮粒度较大 $(126 \mu \mathrm{m})$, 并且选择了较
大的径向进给量 $f_{\mathrm{r}}=0.5 \mathrm{~mm}$ 。表面粗糙度可通过使用 细粒度砂轮或低进给率得以改善。

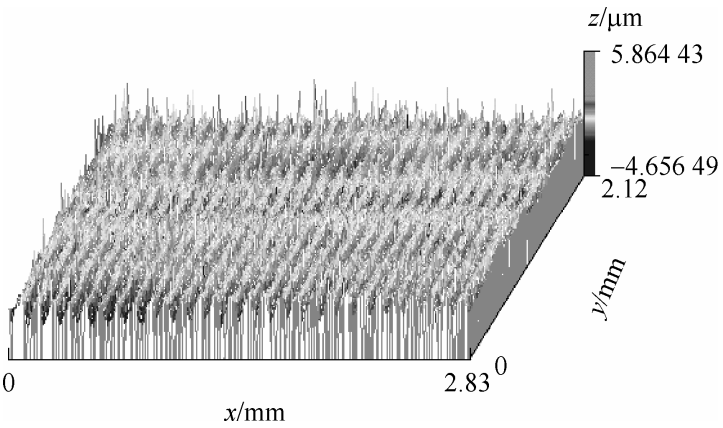

图 14 精密铣磨表面粗粘度及微观形貌特征

以上试验表明: 采用超声振动辅助五轴加工中 心及本文提出的加工方案, 可实现按照非球面方程 直接加工 $\mathrm{SiC}$ 离轴非球面反射镜, 并获得较高的加 工效率和加工精度。对于本文的加工案例, 若沿用 传统工艺按照离轴非球面的最接近球面进行铣磨加 工, 获得的面形误差将大于 $954 \mu \mathrm{m}$ 。如果依靠精密 研磨去除如此大的误差量, 按粗略估算, 至少需要 3 个月的时间才能完成 ${ }^{[16]}$ 。因此本文提供的加工方 法为大幅度缩短 $\mathrm{SiC}$ 反射镜的精密研磨周期、尤其 是为 $1 \mathrm{~m}$ 量级反射镜面形误差的快速收玫, 提供了 解决方案。本文提出的加工方法已经在实际工程应 用中得到多次实践，效果良好。下一步将开展降低 表面粗䊁度及亚表面损伤层厚度等方面的研究。

\section{4 结论}

（1）基于 $\mathrm{CAD} / \mathrm{CAM}$ 软件，开发了离轴非球面 的计算机辅助建模及数控程序自动生成技术。

(2) 提出了五轴联动斜轴定角度加工方式, 实 现了高陡度离轴非球面的高精度铣磨加工。

(3) 提出了旋转中心在镜面外的螺旋走刀方 式，避免了镜面几何中心处的加工残留。

(4) 两次补偿加工后的面形精度 PV 为 $18.8 \mu \mathrm{m}$, $\mathrm{RMS}$ 为 $3.5 \mu \mathrm{m}$ 。

\section{参 考 文 献}

[1] ZHANG Xuejun, CAO Jianlin. Manufacturing of a three mirror anastigmat telescope $[\mathrm{J}]$. Proc. of SPIE, 2003, 4829: $884-885$

[2] WILSON R N. Reflecting telescope optics II : Manufacture, testing, alignment, modern techniques[M]. New York: Springer-Verlag, LLC, 2002.

[3] COMLEY P, MORANTZ P, SHORE P, et al. Grinding metre scale mirror segments for the E-ELT ground based 
telescope[J]. CIRP Annals, Manufacturing Technology, 2011, 60: 379-382.

[4] TONNELLIER X. Precision grinding for rapid manufacturing of large optics[D]. England: Cranfield University, 2009.

[5] 李洁, 雷向阳, 钟波, 等. 大口径方形非球面镜的高效 磨削技术研究 $[\mathrm{J}]$. 金刚石与磨料磨具工程, 2011, 2(31): 74-78.

LI Jie, LEI Xiangyang, ZHONG Bo, et al. Research of high efficiency grinding technology for large square aspheric lens[J]. Diamond \& Abrasives Engineering, 2011, 2(31): 74-78.

[6] KANG Nianhui, LI Shengyi, ZHENG Ziwen. Research on geometric model of grinding large and medium scales optical aspheric surfaces[J]. Proc. of SPIE, 2006, 6034, 60341B-1-60341B-7.

[7] 康念辉, 李圣怡, 郑子文. 基于多体系统理论的非球面 磨削误差模型与补偿技术 $[\mathrm{J}]$. 机械工程学报, 2008, 44(4): 143-149.

KANG Nianhui, LI Shengyi, ZHENG Ziwen. Error model and compensation technology of aspheric grinding based on multibody system theory[J]. Chinese Journal of Mechanical Engineering, 2008, 44(4): 143-149.

[8] 张学军, 李志来, 张忠玉. 基于 $\mathrm{SiC}$ 材料的空间相机 非球面反射镜结构设计 [J]. 红外与激光工程, 2007 , 36(5): 577-582.

ZHANG Xuejun, LI Zhilai, ZHANG Zhongyu. Space telescope aspherical mirror structure design based on $\mathrm{SiC}$ material[J]. Infrared and Laser Engineering, 2007, 36(5): 577-582.

[9] MALACARA D. Optical shop testing[M]. 3rd ed. New Jersey: Wiley Interscience, 2007.

[10] 房丰洲, 张效栋. 大离轴量离轴非球面镜的一种超精密 车削加工方法: 中国, ZL201010530555.9[P]. 2011-05-11.

FANG Fengzhou, ZHANG Xiaodong. An ultrasonic turning method for machining of large off-axis aspherical mirrors: China, ZL201010530555.9[P]. 2011-05-11.

[11] CHEN Fengjun, YIN Shaohui, OHMORI H, et al. Form error compensation in single-point inclined axis nanogrinding for small aspheric insert[J]. Int. J. Adv. Manuf. Technol., 2010: s00170-012-4182-4.

[12] SHORE P. Grinding mode of the "BOX" ultra precision free-form grinder[J]. ASPE Proceedings, Session VIII, Machining, 2005.

[13] 陈逢军, 尹韶辉, 范玉峰, 等. 一种非球面超精密单点 磨削与形状误差补偿技术 $[\mathrm{J}]$. 机械工程学报, 2010, 46(23): 186-191.

CHEN Fengjun, YIN Shaohui, FAN Yufeng, et al. Ultra-precision single-point grinding technique and profile error compensation method for machining aspheric mould[J]. Journal of Mechanical Engineering, 2010, 46(23): 186-191.

[14] MORI M, FUJISHIMA M, YOHEI O. 5 axis mill turn and hybrid machining for advanced application[J]. Procedia. CIRP, 2012, 1: 22-27.

[15] TONNELLIER X, SHORE P, MORANTZ P, et al. Surface quality of a $1 \mathrm{~m}$ Zerodur ${ }^{\circledR}$ part using an effective grinding mode[J]. Proc. of SPIE, 2008, 7102 : 71020B-1-71020B-9.

[16] 李锐钢, 郑立功, 张峰, 等. 大口径高陡度离轴非球面 精磨阶段的数控加工 [J]. 光学精密工程, 2007, 15(5): 633-639.

LI Ruigang, ZHENG Ligong, ZHANG Feng, et al. Computer controlled manufacturing during fine grinding stage of highly steep off-axis asphere with large aperture[J]. Optics and Precision Engineering, 2007, 15(5): 633-639.

作者简介: 张志宇(通信作者), 男, 1980 年出生, 博士, 副研究员。主 要研究方向为硬脆材料超精密切削、微能量电火花放电加工、大口径 $\mathrm{SiC}$ 反射镜精密和超精密铣磨加工。

E-mail: zhangzhiyu@ciomp.ac.cn

李锐钢, 男, 1975 年出生, 博士, 副研究员。主要研究方向为大口径非 球面反射镜加工及检测技术。

E-mail: liruigang@sohu.com

郑立功, 男, 1969 年出生, 博士, 研究员。主要研究方向为超精密加工 及检测技术。

E-mail: zhenglg@ ciomp.ac.cn

张学军, 男, 1968 年出生, 博士, 研究员, 博士研究生导师。主要研究 方向为大口径非球面反射镜加工与检测、空间相机总体设计。

E-mail: zxj@ ciomp.ac.cn 